\title{
Highlights of Results by CMS
}

\author{
Milos Dordevic on behalf of the CMS Collaboration
}

Vinca Institute of Nuclear Sciences, National Institute of the Republic of Serbia, University of Belgrade, Belgrade, Serbia

\begin{abstract}
A selection of the most recent results from the CMS Collaboration at CERN is presented in this overview. The CMS detector status and performance in the LHC Run 2 are outlined. The results of Standard Model physics studies, the studies of the Higgs boson production and properties, as well as a selection of the results of beyond Standard Model searches and an observation of a new excited beauty baryon are presented and discussed. All the results outlined in this overview are found to be in a good agreement with the Standard Model of particle physics. The activities of the CMS Collaboration during the Long Shutdown 2, status and plans for the incoming LHC Run 3, as well as preparation for the HL-LHC are also presented.
\end{abstract}

Keywords: CMS, standard model, Higgs, LHC

DOI: 10.31526/ACP.BSM-2021.12

\section{INTRODUCTION}

The CMS experiment [1] at CERN has conducted a very successful data taking and operation during the LHC Run 2 period. Highlights from Standard Model, Higgs boson physics and beyond Standard Model (SUSY) searches, as well as an observation of a new excited beauty baryon will be presented here. A comparisons will be performed with the prediction of the Standard Model of particle physics for each of the analysis highlighted in this overview. The current status and plans for the CMS detector upgrade in the view of the incoming LHC Run 3 and in preparation for the future High Luminosity HL-LHC will be outlined as a summary.

\section{CMS DETECTOR PHASE-1 UPGRADE AND PERFORMANCE IN LHC RUN 2}

The CMS detector underwent a considerable improvements during the Phase-1 Upgrade [2], including a new hardware installation in the majority of the detector subsystems. These improvements allowed to gain sensitivity of many physics analyses within CMS.

During the LHC Run 2 the CMS detector recorded around $140 \mathrm{fb}^{-1}$ of highest quality data. One of the greatest challenges in data analysis is the effect of pileup. The Fig. 1 shows a distribution of the mean number of inelastic interactions per crossing (pileup) during the LHC Run 2. At CMS, a dedicated algorithm is used to suppress the effect of pileup, in particular for the jets and missing transverse energy reconstruction, that is called PUPPI (Pile Up Per Particle ID) [3]. The PUPPI algorithm uses a distribution of neighbouring particles to estimate the probability of neutral particles to originate from pileup. It was widely used during the Run 2 and PUPPI is now becoming a default for the Run 3. In recent years there has been a lot of development and usage of the Deep Neural Networks (DNN) at CMS, mostly for the jet reconstruction, but also for the reconstruction of tau leptons and for b-tagging. An example of the performance gain in the identification of hadronically decaying W boson using the DeepAK8 jet algorithms [4] with respect to the standard jet taggers is demonstrated by showing the receiver operating characteristic (ROC) curve in the Fig. 2.

\section{HIGHLIGHTS OF STANDARD MODEL RESULTS}

In the following section, a selection of some of the most recent Standard Model physics results from the CMS Collaboration is highlighted.

\subsection{Wr production and EFT constraints}

This is a measurement of the fiducial cross section for the $W \gamma$ production [5], based on the full LHC Run 2 dataset and interpreted within the framework of the Effective Field Theory (EFT). This very fundamental Standard Model process is sensitive and can probe the $W W \gamma$ triple gauge couplings (TGC) that could be altered in new physics scenarios with alternate symmetries or symmetry breaking mechanisms. The signal events are represented with either an electron or a muon produced in the association to the isolated photon. Multiple processes can contribute as a background to such a signal, either the prompt leptons or prompt photons, including the $Z \gamma, t t+\gamma$ and diboson $+\gamma$ events. The signal is extracted by performing a simultaneous binned maximum likelihood fit to the $m_{l \gamma}$ invariant mass distribution, represented in Fig. 3. The measured production cross sections are found to be in a good agreement with the MadGraph5_aMC@NLO and POWHEG predictions. The photon transverse momentum distribution, shown in Fig. 4, is used to extract the expected and observed 95\% CL limits on the four operators in the EFT framework. The limits on $c_{W W W} / \Lambda^{2}$ are 1.75 times lower comparing to the previous best results. These are the most stringent limits to date on this coefficient. 


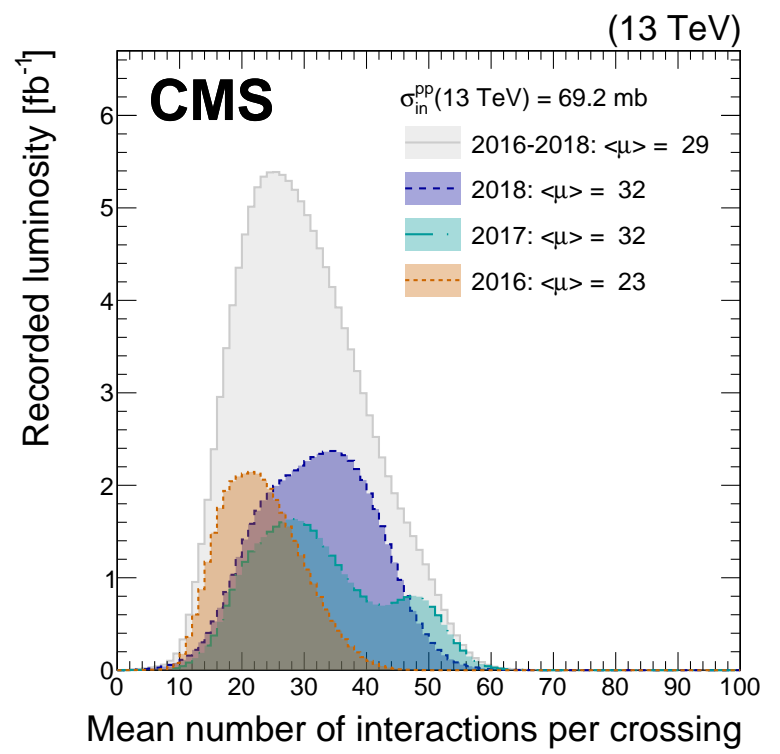

FIGURE 1: Distribution of the mean number of inelastic interactions per crossing (pileup) in data for pp collisions in 2016 (orange), 2017 (light blue), and 2018 (navy blue). [3].

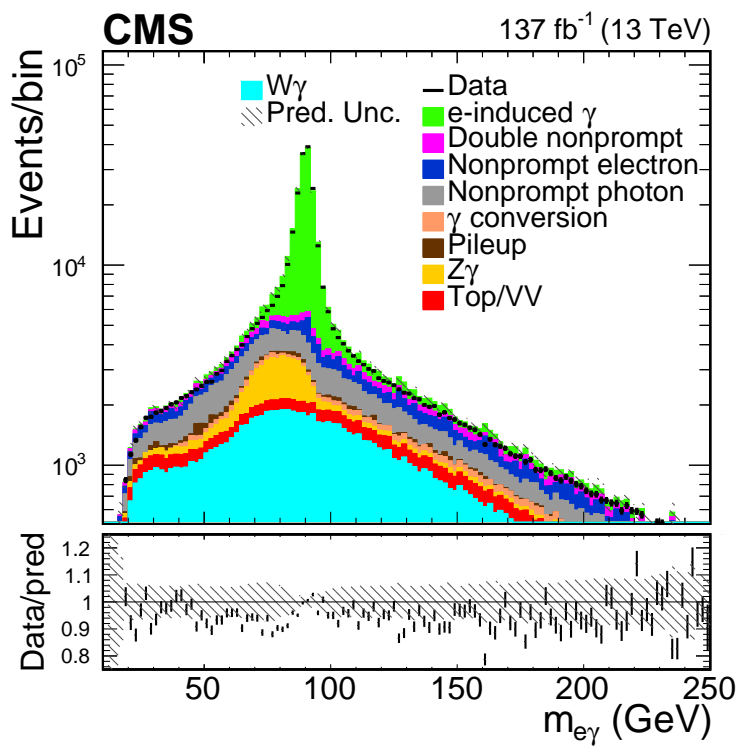

FIGURE 3: Expected and observed distributions in the invariant mass of the lepton-photon system, shown in the electron channel. [5].

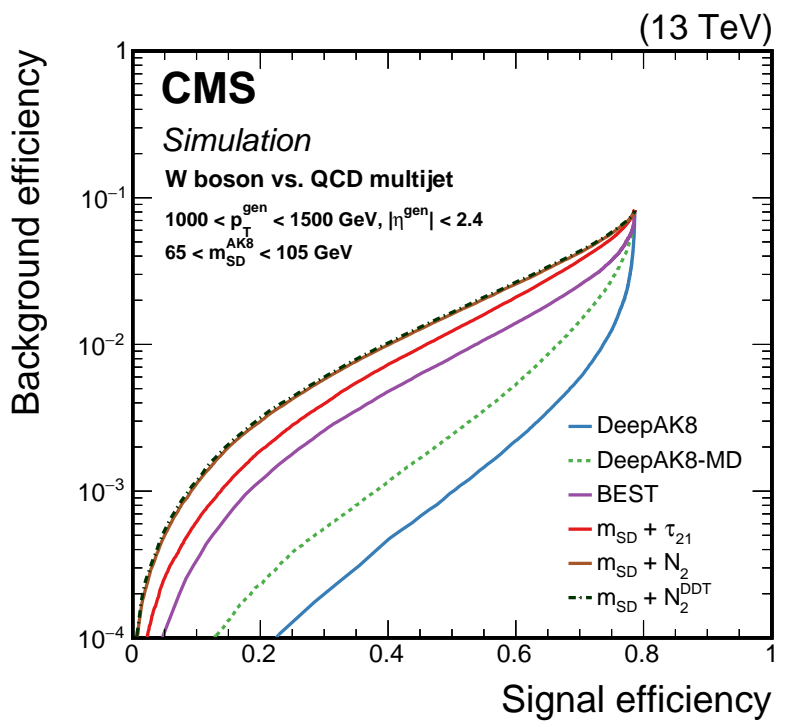

FIGURE 2: Comparison of the identification algorithms for the hadronically decaying $\mathrm{W}$ boson in terms of ROC curves, shown in region of $1000<p_{T}<1500 \mathrm{GeV}$.) [4].

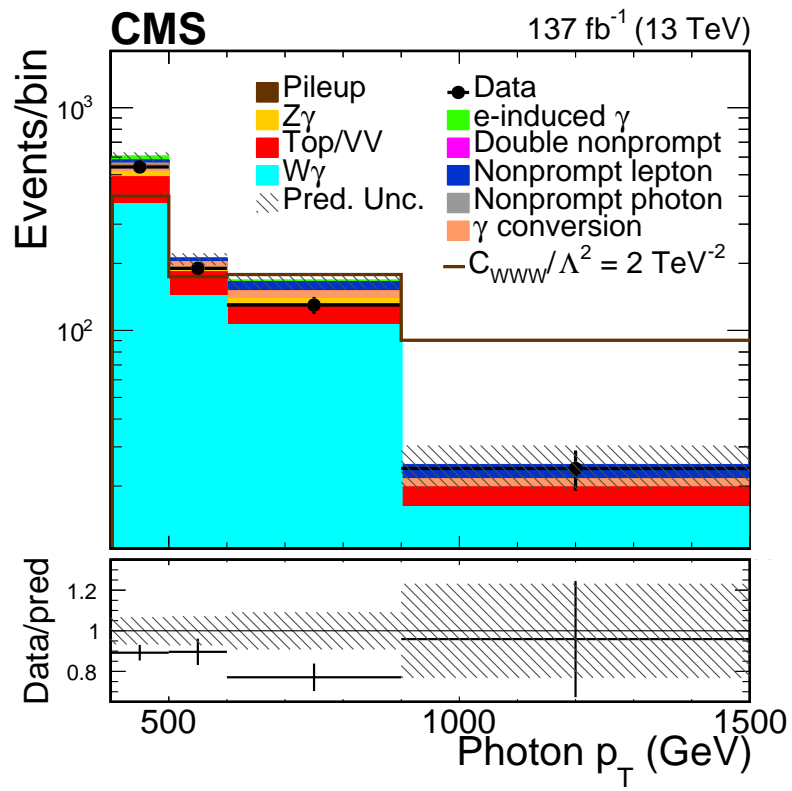

FIGURE 4: The photon $p_{T}$ distribution used for the extraction of limits on dimension-six EFT operators. [5].

\subsection{Top pair plus charm jets production}

This is a first measurement [6] to date of the top pair production with additional charmed jets, using the CMS data collected in 2017. It is much harder to experimentally distinguish $c$ than $b$ jets from light-flavour (LF) or gluon jets. With the development of a charm jet identification algorithm ("c tagger") [7] these signatures can now be disentangled. Since the tt+bb events can originate also from the $\mathrm{tt}+$ Higgs $(\mathrm{ttH})$ process where Higgs boson decays to a pair of bottom quarks, the $\mathrm{tt}+\mathrm{bb}$ and also the $\mathrm{tt}+\mathrm{cc}$, due to $\mathrm{c}$ quark misidentification, represent a non-negligible background to the $\mathrm{ttH}$ process. For this reason, this measurement provides a basis for an estimate of the $\mathrm{tt}+\mathrm{cc}$ background for the future $\mathrm{ttH}$ studies. A new charm-jet identification based on DNN is introduced, trained for the correct jet-parton assignment. A template fitting method is used, based on the outputs of the neural network classifier, in order to identify the signal categories defined by the flavour of the additional jets. This allows a simultaneous extraction of the cross section for $\mathrm{tt}+\mathrm{cc}, \mathrm{tt}+\mathrm{bb}$ and $\mathrm{tt}+\mathrm{LL}$ processes. The $\mathrm{t}+\mathrm{cc}$ cross section was measured in the fiducial phase space, closely matching the sensitive regions of the detector and also in the full phase space. For the first time the ratios of these cross sections were measured, both in fiducial and full phase space, being in a good agreement with respect to the prediction of MadGraph5_aMC@NLO and 
POWHEG generators. The agreement can be also quantified to the level of one to two standard deviations between the measured values and theoretical predictions, shown in the two-dimensional likelihood scans in the Fig. 5.
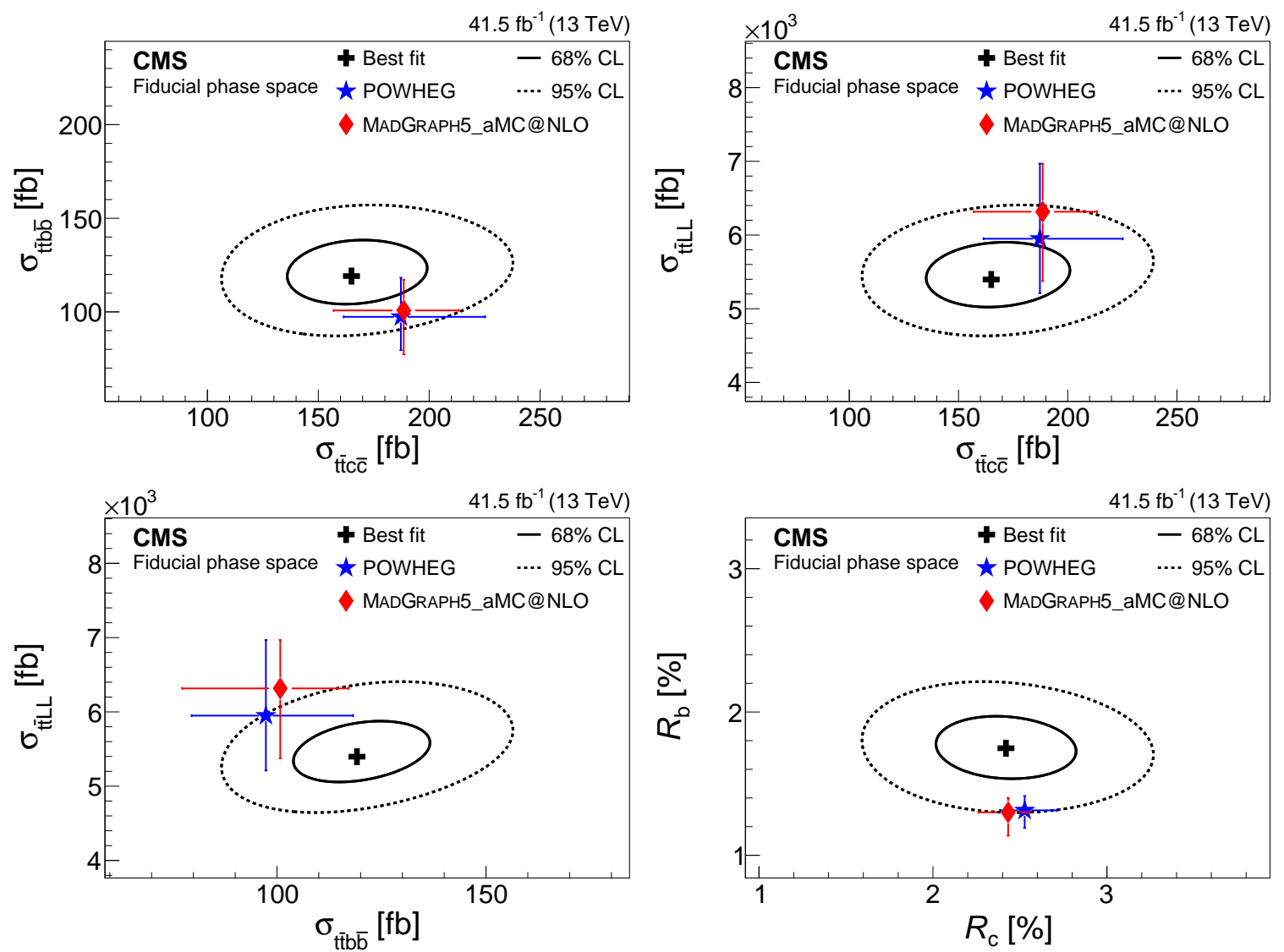

FIGURE 5: Results of the two-dimensional likelihood scans for several combinations of the parameters of interest in the fiducial phase space. [6].

\section{HIGHLIGHTS OF HIGGS BOSON STUDIES}

In the following section, a selection of some of the most recent Higgs boson physics studies from the CMS Collaboration is highlighted.

\subsection{Higgs boson decay to pair of muons}

This is a measurement [8] of the Higgs boson decay to a pair of muons, using the full LHC Run 2 dataset. It is the most accessible probe of the Higgs boson coupling to the second generation fermions at the LHC. Here the first evidence for the existence of this process, with a significance of the observed (expected) 3.0 (2.5) standard deviations, is reported, assuming the most precise measurement of the Higgs boson mass to date, having a value of $m_{H}=125.38 \pm 0.14 \mathrm{GeV}$ [9]. The events are separated into mutually exclusive classes, targeting the main Higgs production modes. For the Vector Boson Fusion (VBF) channel, a DNN is trained using the dimuon and dijet kinematic information, while for the other channels Boosted Decision Trees have been employed. Fig. 6 presents the weighted dimuon invariant mass distribution for all event categories. A new point on the graph showing the coupling strength versus the fermion mass has been added, corresponding to muon coupling to the Higgs boson, as shown in Fig. 7. This new point is in an agreement with the linear dependence of the coupling to the mass, as predicted by the Standard Model. The signal strength modifiers were measured for each production mode and agree well with the Standard Model expectation. The combined signal strength was found to have a value of 1.19. The combination of the results of this analysis with the corresponding analyses at 7 and $8 \mathrm{TeV}$ centre-of-mass energy was performed, resulting in an overall sensitivity improvement of an order of $1 \%$.

\subsection{Higgs boson production with a pair of top quarks}

The $\mathrm{ttH}$ production was observed in 2018 [10] by the CMS Collaboration, in the analysis that used a combination of several final states. This process allows for a direct measurement of the magnitude of the Yukawa top coupling that in the Standard Model is at the order of unity. The single top quark production associated to the Higgs boson $(\mathrm{tH})$ is a process that can give an information about the sign of this coupling. This measurement [11], based on the full LHC Run 2 dataset, provides a sensitivity to the ttH 
$137 \mathrm{fb}^{-1}(13 \mathrm{TeV})$

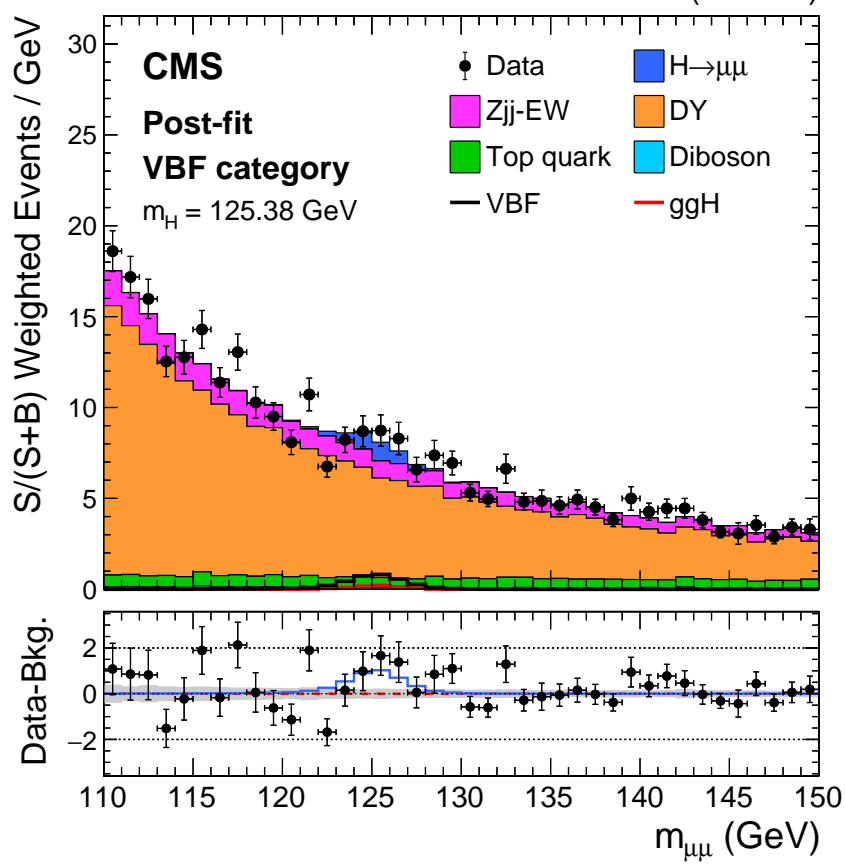

FIGURE 6: The $m_{\mu \mu}$ distribution for the weighted combination of VBF-SB and VBF-SR events. [8].

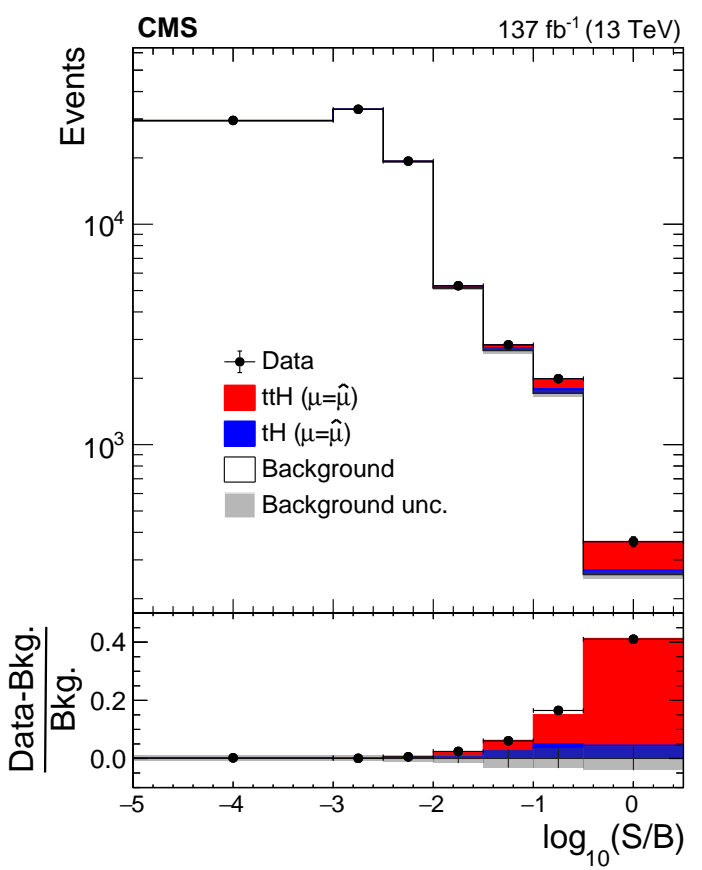

FIGURE 8: Distribution of the decimal logarithm of the ratio between the expected $\mathrm{ttH}+\mathrm{tH}$ signal and the expected sum of background contributions in each bin of the 105 distributions that are included in the maximum likelihood fit used for the signal extraction. [11].

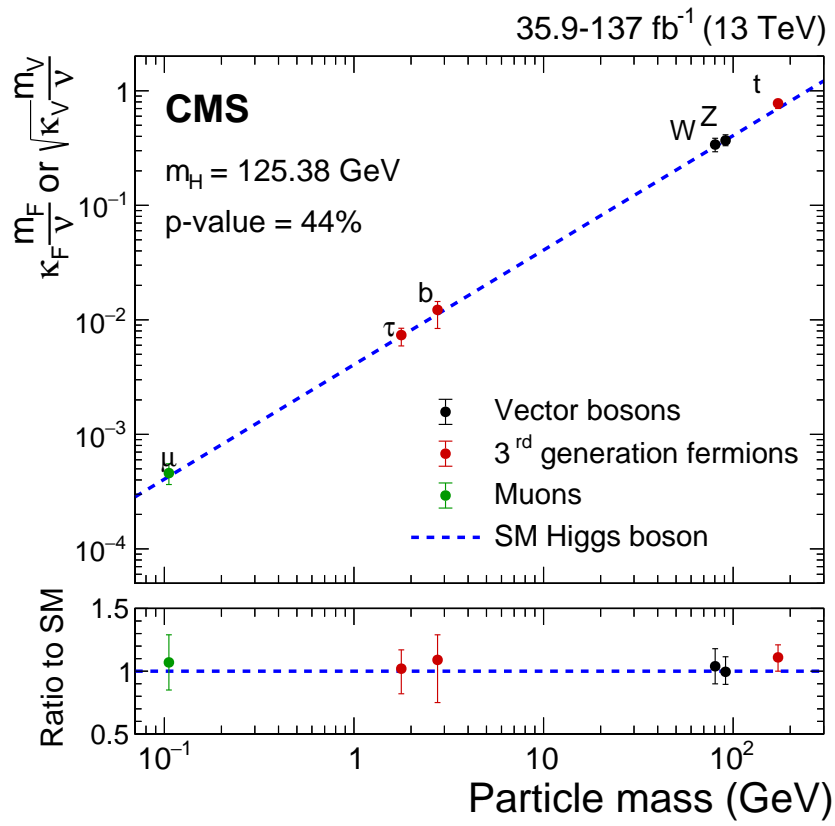

FIGURE 7: The best fit estimates for the reduced coupling modifiers extracted for fermions and weak bosons from the resolved $\kappa$-framework compared to their corresponding prediction from the SM. [8].

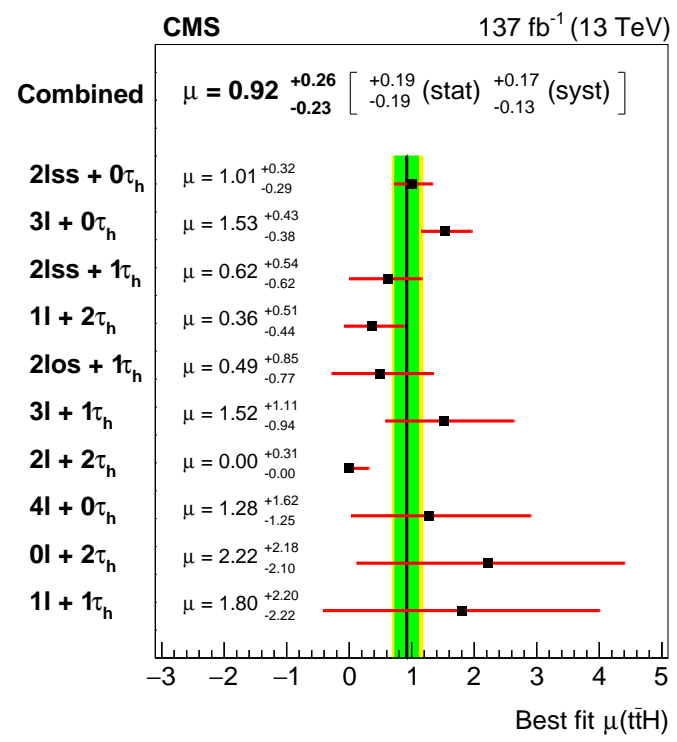

FIGURE 9: Production rate of the $\mathrm{ttH}$ signal, in units of their rate of production expected in the SM, measured in each of the ten channels individually and for the combination of all channels. [11].

production with an observed (expected) 4.7 (5.2) significance, using only the multilepton channel, where the Higgs boson decays to a pair of $\mathrm{W}$ bosons, to a pair of $\mathrm{Z}$ bosons or to a pair of $\tau$ leptons. Both the $\mathrm{ttH}$ and $\mathrm{tH}$ processes were studied in this measurement and the signal yield was maximized using ten different signatures based on the lepton multiplicity in the final state. In order to separate the $\mathrm{tH}$ and $\mathrm{ttH}$ signal from the sizeable residual background, a maximum likelihood fit has been applied to a number of discriminating variables that are the output of the machine-learning algorithms, multiclass ANN and DNN, trained using tH and 

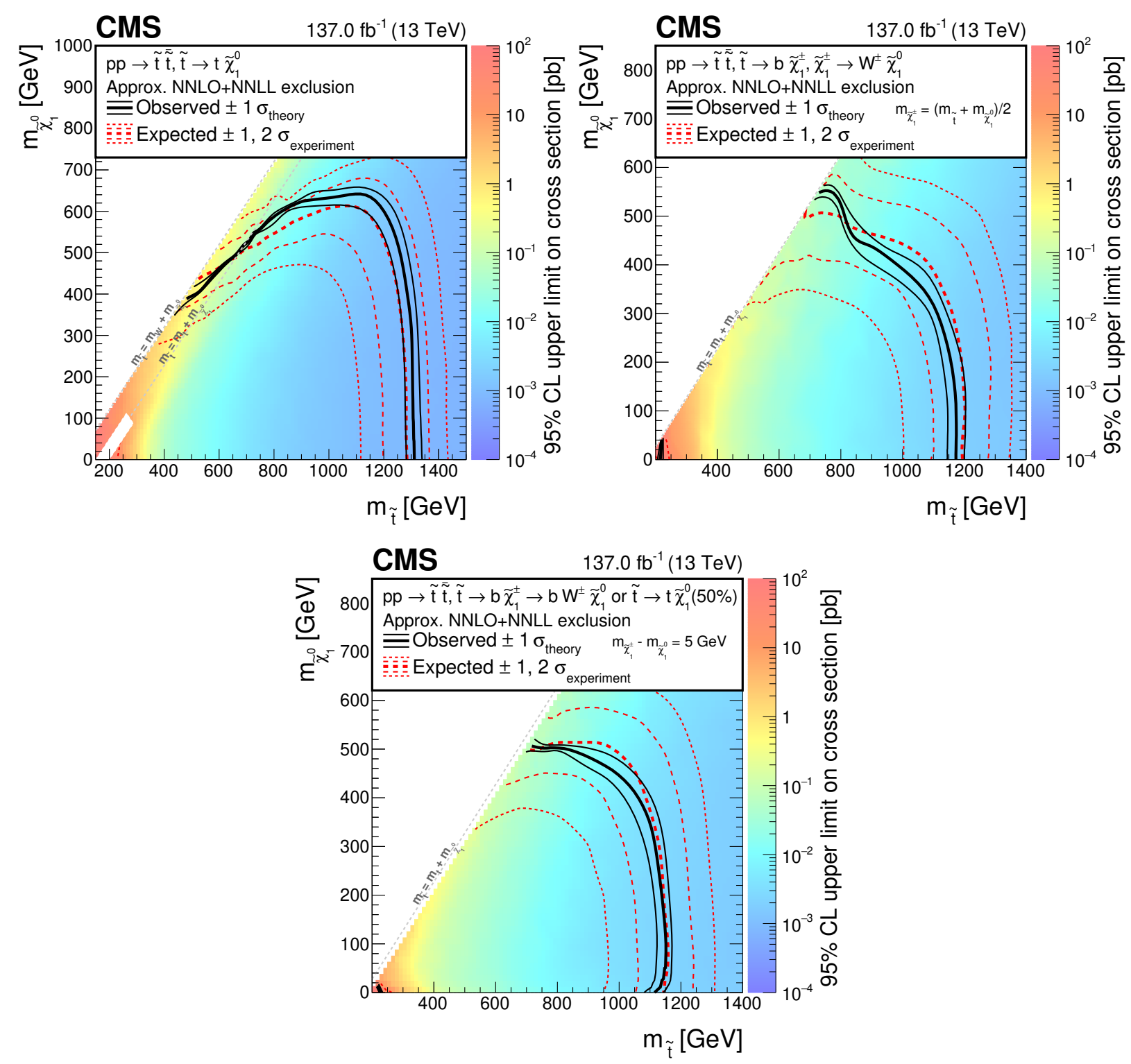

FIGURE 10: The 95\% CL upper limit on the production cross section of the T2tt (upper left), T2bW (upper right), and T2tb (lower) simplified models as a function of the top squark and LSP masses. [12].

$\mathrm{ttH}$ events against the leading background processes. In Fig. 8 there is a combined distribution showing the expected $\mathrm{tH}$ and $\mathrm{ttH}$ signal contributions and the expected sum of backgrounds, while in the Fig. 9 the $\mathrm{ttH}$ signal strength was presented for each of the ten channels individually, as well as for the combination of all channels. The Yukawa coupling of the Higgs boson to the top quark was constrained within the $-0.9<y_{T}<-0.7$ and $0.7<y_{T}<1.1$ times the Standard Model expectation, at the $95 \%$ confidence level.

\section{HIGHLIGHTS OF BEYOND STANDARD MODEL SEARCHES}

In the following section, one of the most recent searches for supersymmetric particles from the CMS Collaboration is presented.

\subsection{Fully-hadronic top squark production}

This measurement [12] represents a search for the supersymmetric partners of the top quarks, the top squark, performed in the fully hadronic final state. In such final state there are multiple jets, no leptons and large missing transverse energy in the event. The measurement is performed using the full LHC Run 2 dataset. The targeted production scenarios are the direct and gluinomediated top squark production. The top quark and $\mathrm{W}$ boson tagging algorithms were applied for both merged, i.e. where at high momentum the decay products of a hadronically decaying $\mathrm{W}$ boson or top quark tend to merge to a single jet, and resolved, where at low momentum hadronic top decays can be resolved into three jets. Dedicated DNNs were trained in order to select 

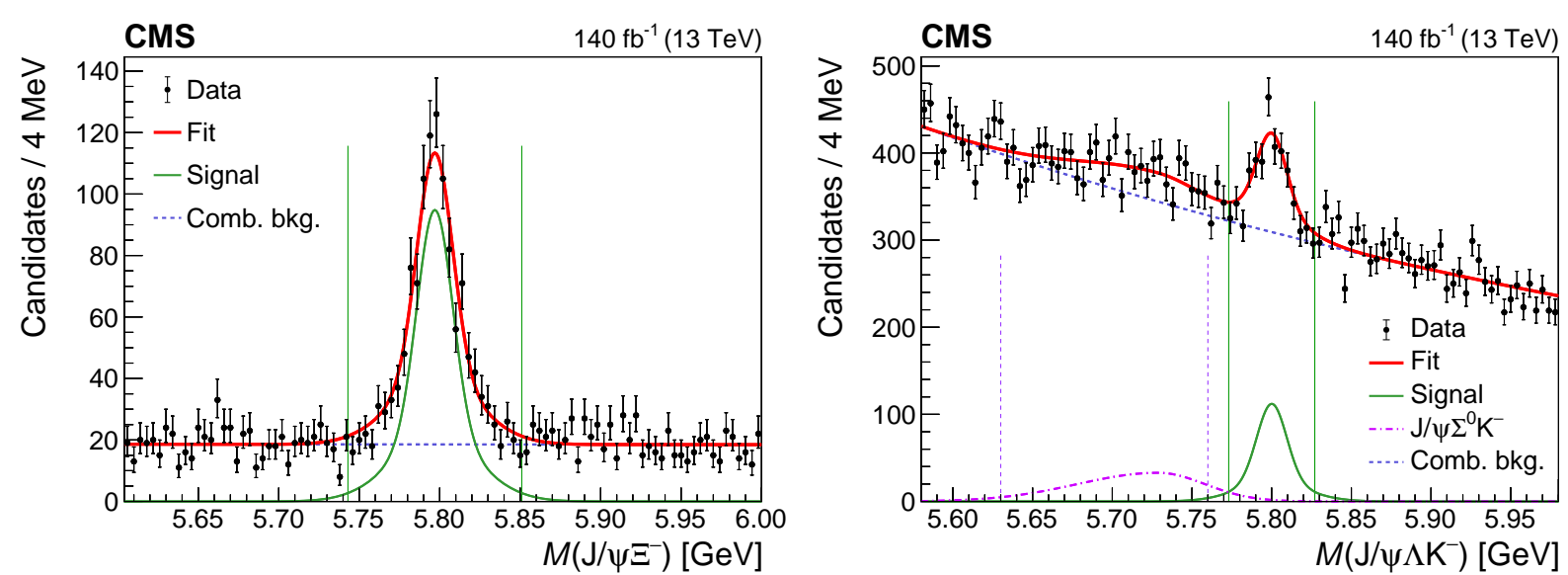

FIGURE 11: Invariant mass distributions of the selected $\Xi_{b}$ candidates in the $J / \psi \Xi^{-}$(left) and $J / \psi \Lambda K^{-}$(right) decay channels with the fit results superimposed. [14].

each of these signatures. The multiclass DeepAK8 algorithm [4] is used for the boosted top quark and boosted W boson, while the DeepResolved algorithm [13] was applied for the reconstruction of the top quark with low transverse momentum. In case of the direct top squark production, two search regions are defined, based on the value of the delta mass variable, defined as a difference between the top squark mass and the mass of the LSP particle. One search region corresponds to the delta mass higher than the mass of the $\mathrm{W}$ boson, while the other search region is reserved for the delta mass lower than the $\mathrm{W}$ boson mass. These two regions are further kinematically divided into a total of 183 search bins. All but six out of 183 search bins have agreement within two standard deviations, and all search bins have agreement within three standard deviations. The 95\% confidence level upper limits are established on the cross section for several simplified models of direct and gluino-mediated top squark production, as a function of masses of the supersymmetric particles. For the direct production the top squark masses are excluded below a limit ranging from 1150 to $1310 \mathrm{GeV}$ in the region of parameter space where the delta mass variable is larger than W boson mass and below a limit ranging from 630 to $750 \mathrm{GeV}$ for the delta mass smaller than $\mathrm{W}$ mass. These limits are shown in Fig. 10, representing the most stringent constraints on these models to date. In the case of gluino-mediated production, the gluino masses are excluded below a limit ranging from 2150 to $2260 \mathrm{GeV}$, depending on a signal model. In summary, these results significantly extend the previous fully hadronic top squark searches by 100 to $300 \mathrm{GeV}$ due to four times larger dataset used and improved analysis methods.

\section{OBSERVATION OF A NEW BARYON $\Xi_{B}(6100)^{-}$}

This measurement [14] represents the observation of a new excited beauty baryon, the $\Xi_{b}(6100)^{-}$, performed using the full LHC Run 2 dataset. Two fully reconstructed states are considered, a first one where the ground state $\Xi_{b}$ decays to a $J / \Psi_{\text {and }} \Xi^{-}$and a second one where it decays to a $J / \Psi, \Lambda$ and $K^{-}$, that are each followed by their corresponding decays. There is also one partially reconstructed state of the decay to $J / \Psi, \Sigma^{0}$ and $K^{-}$, with the $\Sigma^{0}$ decaying to a $\Lambda$ and a photon that is too soft to be detected. The invariant mass of the $\Xi_{b}$ is shown in Fig. 11 for the two fully reconstructed cases, where on the left plot one can see a fitted signal, shown with green line, described by a double-Gaussian and a background described by a first order polynomial, while on the right plot the background model is an exponential function. The fitted yield of a partially reconstructed state is shown with dotteddashed curve. The analysis is performed using the mass difference variable, defined as indicated in the x-axis of the Fig. 11, that has a better mass resolution than the invariant mass and is also insensitive to a potential mass shift caused by the emitted photon. The resulting signal significance varies between 6.2 and 6.7 sigma, depending on the fit model variations used to evaluate the systematic uncertainties and which represents an observation of this particle. The mass was measured using the mass difference formula and is found to have a value of $6100.3 \pm 0.2$ (stat.) \pm 0.1 (syst.) $+0.6\left(\Xi_{b}^{-}\right) \mathrm{MeV}$. The natural width of this resonance is compatible with zero and a $95 \%$ confidence level upper limit of $1.9 \mathrm{MeV}$ has been determined.

\section{CMS ACTIVITIES DURING LONG SHUTDOWN 2 AND PLANS FOR LHC RUN 3}

Following the end of LHC Run 2 in 2018, the CMS detector is presently in its maintenance and upgrade phase, call the Long Shutdown 2. Only a small delay with respect to the original schedule has been accumulated during the previous 12 months and the data taking is foreseen to restart in 2022. The activities related to the CMS Phase 2 Upgrade are taking place already and will be in time for the first scheduled test beams in 2022. The CMS Collaboration plans for the Run 3 data taking are not only to collect a dataset with larger statistics, but also to improve on the analysis methods, in particular when it comes to usage of DNNs that are expected to provide an enhanced signal to background ratio for many physics analysis. The upgrades to highlight are the new Pixel detector with updated Layer-1 electronics, the depth segmentation from the new readout in HCAL detector, the newly installed GEM detector, as well as the heterogeneous computing architecture that is being implemented at the HLT, being now a mixture of 

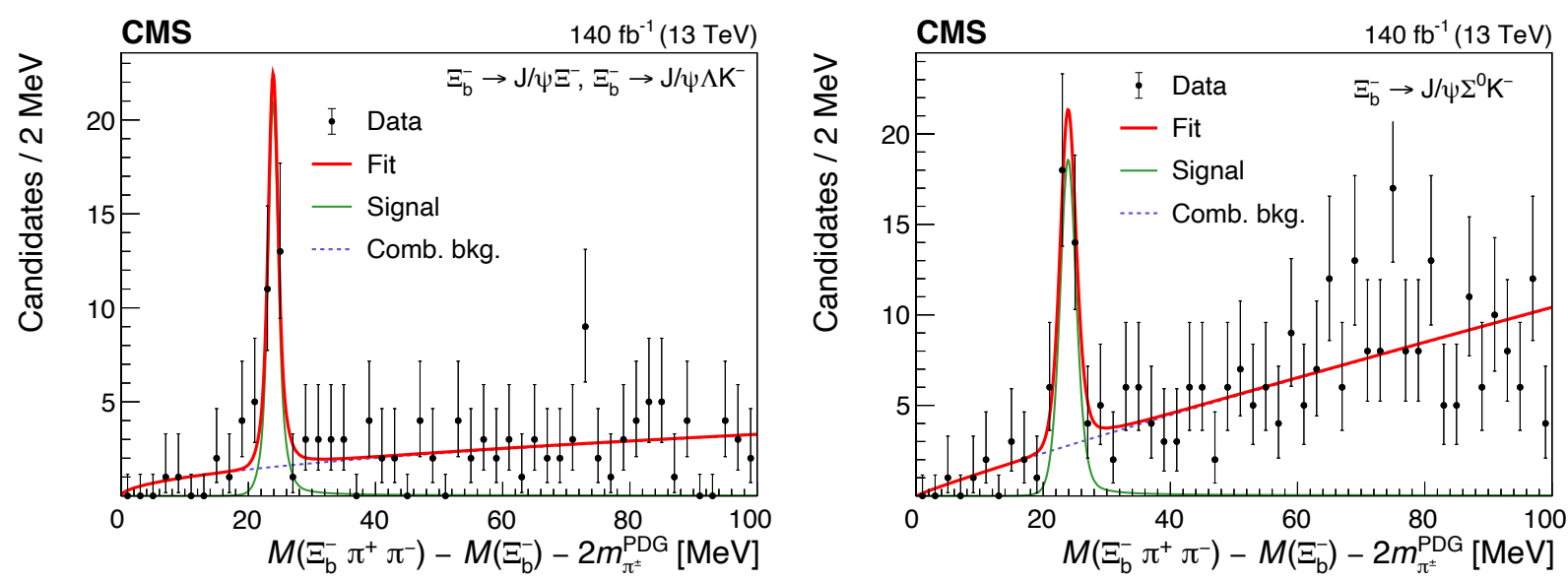

FIGURE 12: Distributions of the invariant mass difference $\Delta \mathrm{M}$ for the selected $\Xi_{b}^{-} \pi^{+} \pi^{-}$candidates, with the $\Xi_{b}$ reconstructed in the $J / \psi \Xi^{-}$and $J / \psi \Lambda K^{-}$channels or partially reconstructed in the $J / \psi \Sigma_{0} K^{-}$channel (right). [14].

CPU and GPU, in contrast to the previous sole usage of the CPUs at the HLT, tested to yield a $25 \%$ reduction in HLT CPU timing already.

\section{SUMMARY AND OUTLOOK}

The CMS Collaboration has produced many important new results, exploiting the full LHC Run 2 dataset. In this overview, a selection of the most recent results of Standard Model measurements, Higgs boson studies and beyond Standard Model searches are presented, together with a report on the observation of a new beauty strange baryon. A large number of analyses in CMS Collaboration is using novel machine learning algorithms like DNNs which are also used for object reconstruction and Trigger. Many exciting physics results will be produced with the expected $300 \mathrm{fb}^{-1}$ in LHC Run $2+$ Run 3 and $3000 \mathrm{fb}^{-1}$ planned for the HL-LHC.

\section{References}

[1] The CMS Collaboration, JINST 3 S08004 (2008).

[2] Technical proposal for the upgrade of the CMS detector through 2020, https://cds.cern.ch/record/1355706.

[3] The CMS Collaboration, JINST 15 (2020) P09018.

[4] The CMS Collaboration, JINST 15 (2020) P06005.

[5] The CMS Collaboration, arXiv:2102.02283, Submitted to Phys. Rev. Lett.

[6] The CMS Collaboration, arXiv:2012.09225, Submitted to Phys. Lett. B.

[7] The CMS Collaboration, JINST 13 (2018) P05011.

[8] The CMS Collaboration, JHEP 01 (2021) 148.

[9] The CMS Collaboration, Phys. Lett. B 805 (2020) 135425.

[10] The CMS Collaboration, Phys. Rev. Lett. 120, 231801 (2018).

[11] The CMS Collaboration, arXiv:2011.03652, Submitted to EPJC.

[12] The CMS Collaboration, arXiv:2103.01290, Submitted to Phys. Rev. D.

[13] The CMS Collaboration, JHEP 05 (2020) 032.

[14] The CMS Collaboration, arXiv:2102.04524, Submitted to Phys. Rev. Lett. 